\title{
Thermal-fluid transport phenomena in an axially rotating flow passage with twin concentric orifices of different radii
}

\author{
Shuichi Torii ${ }^{1, *, \dagger}$ and Wen-Jei Yang ${ }^{2}$ \\ ${ }^{1}$ Department of Mechanical Engineering and Materials Science, Kumamoto University, Kumamoto 860-8555, Japan \\ ${ }^{2}$ Mechanical Engineering and Applied Mechanics, The University of Michigan, Ann Arbor, MI 48109, U.S.A.
}

\begin{abstract}
SUMMARY
This paper investigates the thermal fluid-flow transport phenomena in an axially rotating passage in which twin concentric orifices of different radii are installed. Emphasis is placed on the effects of pipe rotation and orifice configuration on the flow and thermal fields, i.e. both the formation of vena contracta and the heattransfer performance behind each orifice. The governing equations are discretized by means of a finitedifference technique and numerically solved for the distributions of velocity vector and fluid temperature subject to constant wall temperature and uniform inlet velocity and fluid temperature. It is found that: (i) for a laminar flow through twin concentric orifices in a pipe, axial pipe rotation causes the vena contracta in the orifice to stretch, resulting in an amplification of heat-transfer performance in the downstream region behind the rear orifice, (ii) simultaneously the heat transfer rate in the area between twin orifice is intensified by pipe rotation, (iii) the amplification of heat transfer performance is affected by the front and rear orifice heights. Results may find applications in automotive and rotating hydraulic transmission lines and in aircraft gas turbine engines. Copyright (C) 2005 John Wiley \& Sons, Ltd.
\end{abstract}

KEY WORDS: secondary flow; rotational effect; vena contracta; numerical analysis; heat transfer; orifice

\section{INTRODUCTION}

If sharp corners or ribs exist in a pipe, flow separation occurs at the corners and a vena contracta is formed. The fluid must accelerate locally to pass through the reduced flow area at the vena contracta, whose minimum area results when the converging streamlines begin to expand to fill the downstream region. This phenomenon is observed in a typical orifice meter, which has been popularly used to measure the instantaneous flow rate in pipes. The other two most common devices for flow-rate measurement are the nozzle meter and the venturi meter. In many practical engineering applications, the working fluid in rotating machinery flows in sudden expansion or contraction passages or in passage with an orifice. The present study is

\footnotetext{
*Correspondence to: Shuichi Torii, Department of Mechanical Engineering and Materials Science, Kumamoto University, Kumamoto 860-8555, Japan.

†E-mail: torii@mech.kumamoto-u.ac.jp
}

Copyright (C) 2005 John Wiley \& Sons, Ltd.

Received 1 October 2004

Revised 11 September 2005 Accepted 9 October 2005 
focused on the secondary flow and heat-transfer performance in an axially rotating pipe with concentric orifices.

Yamada and Imao (1980) measured the streamwise velocity and hydraulic loss in a saturated downstream region of an axially rotating pipe without ribs in which a fully-developed laminar flow is introduced. It was disclosed that the hydraulic loss and the flow instability are induced due to the pipe rotation and that as its speed is increased, the radial distribution of the streamwise velocity approaches the turbulence case. Velocity profiles in the developing flow region of a rotating pipe were analysed by Lavan et al. (1969), Mizutani et al. (1987) and Imao et al. (1989). In particular, Yamada et al. (1985) observed that when the laminar flow enters the axially rotating pipe, a destabilization of the flow due to the swirl is induced, resulting in an enhancement in the turbulence intensity. Torii and Yang (1998a,b, 2000) investigated the thermal and fluid-flow transport phenomena in axially rotating pipes with sudden expansion, contraction, and concentric orifice. In general, industrial rotating machinery has many orifices or ribs in its passage. To further understand the transport phenomena in such passage, detailed information on the heat transfer and fluid flow in the vena contracta is of great importance to many engineering applications. Although numerical simulation provides detailed information on the transport phenomena, the existing theoretical and experimental study is confined to the thermal fluid flow in sudden expansion or contraction passage or in passage with an orifice. Thus no information on these fluid flow transport characteristics gives the design of the industrial applications, because the passage with many sudden expansion- or contraction-areas or the passage with many orifices appears in automotive and rotating hydraulic transmission lines and in aircraft gas turbine engines.

This paper treats the thermal and fluid-flow transport phenomena in an axially rotating pipe in which twin concentric orifices of different heights are installed. Emphasis is placed on the effects of pipe rotation rate and orifice-shape on both the formation of vena contracta and the local heat-transfer rate in the axially rotating passage. A numerical method is employed to determine velocity and temperature profiles.

\section{GOVERNING EQUATIONS AND NUMERICAL METHOD}

Consider a forced flow through an axially rotating pipe with twin concentric orifices, which is heated with uniform wall temperature. The physical configuration and the cylindrical coordinate system of the flow are shown in Figure 1. The following assumptions are imposed in the formulation of the problem based on the characteristics of the flow: it is an incompressible, laminar, steady flow with constant fluid properties; there is constant wall temperature, uniform inlet velocity, and uniform inlet fluid temperature and negligible axial conduction (due to the high Peclet number). Then, the governing differential equations for mass, momentum and energy can be expressed as

Continuity equation:

$$
\frac{\partial U_{i}}{\partial x_{i}}=0
$$

Momentum equation:

$$
U_{j} \frac{\partial U_{i}}{\partial x_{j}}=-\frac{1 \partial P}{\rho \partial x_{i}}+v \frac{\partial}{\partial x_{j}}\left(\frac{\partial U_{i}}{\partial x_{j}}\right)
$$




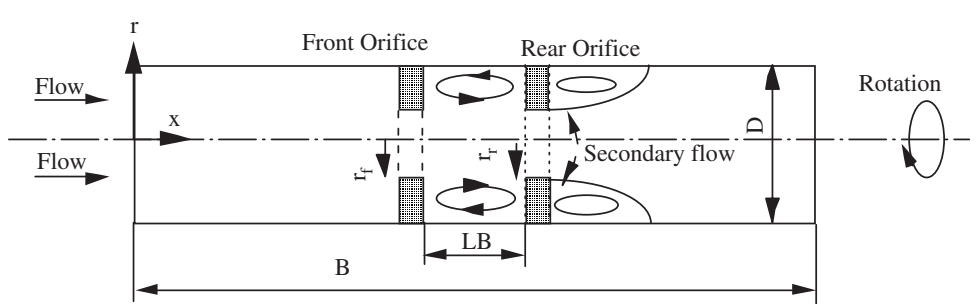

Figure 1. Schematic diagrams of axially rotating pipe flow systems with twin orifices and their co-ordinates.

Energy equation:

$$
U_{i} \frac{\partial T}{\partial x_{i}}=\alpha \frac{\partial}{\partial x_{i}}\left(\frac{\partial T}{\partial x_{i}}\right)
$$

An isothermal laminar flow in the absence of pipe rotation is assumed as the inlet condition. Only one-half of the pipe cross-section is treated because of the symmetry of the fluid flow. The boundary conditions in the axially rotating pipe are specified as

$$
\begin{aligned}
& U=V=0, \quad W=W_{\mathrm{w}}, \quad T=T_{\mathrm{w}} \quad \text { at wall } \\
& U=U_{\text {in }}, \quad V=0, \quad W=0, \quad T=T_{\text {in }} \text { at the inlet, i.e. } x=0 \\
& \frac{\partial U}{\partial r}=0, \quad V=W=0, \quad \frac{\partial T}{\partial r}=0 \quad \text { at centre }(r=0)
\end{aligned}
$$

No-slip boundary condition is applied at the insulated orifice surface. At the exit, the boundary conditions for the dependent variables are obtained by setting the first derivatives in the axial direction equal to zero. This is because the same use in other flow conditions is permissible for computational convenience if the outlet boundary is located in a flow region, which is sufficiently far downstream from the region of interest. Notice that the above boundary condition at the exit is strictly valid only when the flow is fully developed. As the boundary condition in the streamwise direction, a fully-developed condition is used, because the computational domain is sufficiently large, as mentioned in the following.

To discretize the governing equations, the finite difference method is employed here. Note that this method is based on the conservative formulation, although the governing equations (1)-(3) are written in non-conservative form. Here, the discretization method employed in the present study uses a finite difference formulation and the discretized forms of the above governing equations read as

$$
\begin{aligned}
U_{i, j}^{n+1} & =U_{i, j}^{n}+\Delta t\left[\frac{1}{\Delta X}\left(P_{i, j}^{n}-P_{i+1, j}^{n}\right)-\mathrm{FUX}^{n}-\mathrm{FUY}^{n}+\mathrm{VISX}^{n}\right] \\
V_{i, j}^{n+1} & =V_{i, j}^{n}+\Delta t\left[\frac{1}{\Delta Y}\left(P_{i, j}^{n}-P_{i, j+1}^{n}\right)-\mathrm{FVX}^{n}-\mathrm{FVY}^{n}+\mathrm{VISY}^{n}\right] \\
T_{i, j}^{n+1} & =T_{i, j}^{n}+\Delta t\left[-\mathrm{FTX}^{n}-\mathrm{FTY}^{n}+\mathrm{VIST}^{n}\right]
\end{aligned}
$$


The subscript and superscript correspond to the cell location and time level, respectively. A detailed description of the above discretization method and $\mathrm{FUX}^{n}, \mathrm{FUY}^{n}, \mathrm{VISX}^{n}, \mathrm{FVX}^{n}, \mathrm{FVY}^{n}$, and VISY ${ }^{n}$ in Equations (4) and (5), are available in the literature (Harlow and Welch 1965; Hirt et al., 1975). $\mathrm{FTX}^{n}, \mathrm{FTY}^{n}$, and $\mathrm{VIST}^{n}$ in Equation (6) can be expressed as

$$
\begin{aligned}
\mathrm{FTX}^{n}= & \frac{1}{4 \Delta X}\left[\left(U_{i, j}^{n}+U_{i, j+1}^{n}\right)\left(T_{i, j}^{n}+T_{i+1, j}^{n}\right)+\alpha\left|U_{i, j}^{n}+U_{i, j+1}^{n}\right|\left(T_{i, j}^{n}-T_{i+1, j}^{n}\right)\right. \\
& \left.-\left(U_{i-1, j}^{n}+U_{i-1, j+1}^{n}\right)\left(T_{i, j-1}^{n}+T_{i, j}^{n}\right)-\alpha\left|U_{i, j}^{n}+U_{i-1, j+1}^{n}\right|\left(T_{i-1, j}^{n}-T_{i, j}^{n}\right)\right] \\
\mathrm{FTY}^{n}= & \frac{1}{4 \Delta Y}\left[\left(V_{i, j}^{n}+V_{i+1, j}^{n}\right)\left(T_{i, j}^{n}+T_{i, j+1}^{n}\right)+\alpha\left|V_{i, j}^{n}+V_{+1 i, j}^{n}\right|\left(T_{i, j}^{n}-T_{i, j+1}^{n}\right)\right. \\
& \left.-\left(V_{i, j+1}^{n}+V_{i+1, j-1}^{n}\right)\left(T_{i, j-1}^{n}+T_{i, j}^{n}\right)-\alpha\left|V_{i, j-1}^{n}+V_{i+1, j-1}^{n}\right|\left(T_{i, j-1}^{n}-T_{i, j}^{n}\right)\right] \\
\operatorname{VIST}^{n}= & \frac{\lambda}{\rho c_{p}}\left[\frac{1}{\Delta X^{2}}\left(T_{i+1, j}^{n}-2 T_{i, j}^{n}+T_{i-1, j}^{n}\right)+\frac{1}{\Delta Y^{2}}\left(T_{i, j+1}^{n}-2 T_{i, j}^{n}+T_{i, j-1}^{n}\right)\right]
\end{aligned}
$$

The non-dimensional factor $\alpha$ in each equation is a weight coefficient and $\alpha=0.5$ is employed here. The system variables $P, U, V$ and $T$ are calculated with a staggered grid proposed by Harlow and Welch (1965). The processes involved in completing one calculation cycle at an arbitrary time-step are as follows: (i) by solving Equations (4)-(6) for $U, V$ and $T$, subject to the appropriate boundary conditions, the new velocities, which involve the values for the contributing pressure and velocities at the precious time-step, are predicted for the entire mesh to be solved; (ii) these velocities are iteratively adjusted to satisfy the continuity equation (1), by making appropriate changes in the cell pressures, that is, each cell in the iteration is considered successively and is given a pressure change that drives its instantaneous velocity divergence to zero (Torii et al., 1998); (iii) when convergence has been reached with a maximum relative change in the values between successive iterations of less than $10^{-4}$, the velocity and pressure fields are determined at the advanced time level and are used as starting values at next time-step.

Computation reveals only a slight difference when the grid system is properly changed from $20 \times 40 \times 500(r, \theta, x)$ to $40 \times 80 \times 1000$, resulting in a grid-independent solution. Hence, a grid system of $20 \times 40 \times 500$ nodal points with uniformly distributed nodal points is employed here to save computation time. Here, a computational domain in the streamwise direction is $B / R=50$. Based on the data set obtained here, visualization of the flow and thermal fields is carried out using a commercially available 2-D graphics software tool.

The numerical computation was performed on a personal computer, and consumed nearly $78 \mathrm{CPU}$ hours using air as the working fluid $(\operatorname{Pr}=0.71)$. The parameters used in the present study are Reynolds number $\mathrm{Re}=480$, velocity ratios $N=0-5$, radius ratio of the front orifice to the pipe $r_{f}^{*}=0.50-0.88$, and radius ratio of the rear orifice to the pipe, $r_{r}^{*}=$ $0.50-0.88$.

Simulations with grids of various degrees of coarseness, as mentioned earlier, were conducted to determine the required resolution for grid-independent solutions. The maximum error was estimated to be about $2 \%$ by comparing the solutions on regular and fine grids with twice the grid points. 


\section{RESULTS AND DISCUSSION}

Figure 2 illustrates local Nusselt numbers in a stationary pipe with two concentric orifices as a function of radius ratios $r^{*}$ (i.e. $r^{*}=r_{r}^{*}$ and $r_{f}^{*}$ ). Note that $r_{r}^{*}$ is the same as $r_{f}^{*}$. Numerical results are obtained for $\operatorname{Re}=480, N=0.0$, and $L B^{*}=2.50 . N=0$ case refers to stationary pipe. For comparison, the well-known correlation (Kays and Crawford, 1983) of laminar heat transfer in the hydrodynamically and thermally fully-developed flow in the absence of pipe rotation and orifices is superimposed in the figure with a solid straight line. It is observed in Figure 2 that (i) the local Nusselt number is substantially higher than that in the thermally and hydrodynamically fully-developed region due to the thermal entrance effect, (ii) in the region between two orifices and in the vena contracta, the local heat-transfer performance varies along the flow and is affected by the orifice height, and (iii) in particular, the local Nusselt number in the vena contracta, for $r^{*}=0.88$, is higher than that for $r^{*}=0.5$ and is gradually diminished in the downstream direction. It is postulated that heat transfer enhancement in the region between two orifices with the same height and in the vena contracta is substantially induced for $r^{*}=0.88$ than for $r^{*}=0.50$ and 0.75 . In general, the pressure drop in the pipe flow is increased for lower radius ratio although heat transfer performance is amplified due to the presence of the orifice than the flow case without it. Thus, heat transfer characteristics is focused on higher radius ratio.

Attempt is made to investigate the effect of combination of radius ratios of twin orifices on heat transfer performance in the region between two orifices and in the vena contracta. Figure 3 illustrates the local Nusselt numbers in a stationary pipe with two concentric orifices at different radius ratios $r^{*}$ as the parameter. Numerical results are obtained at $\operatorname{Re}=480, N=0.0$, and $L B^{*}=2.50$. One observes that: (i) the local Nusselt number is extremely higher than that in the thermally and hydrodynamically fully-developed region because of the thermal entrance effect as mentioned previously, (ii) in the region between two orifices, higher local heat-transfer performance for $r_{f}^{*}=0.75$ and $r_{r}^{*}=0.88$ appears along the flow, and (iii) the local Nusselt

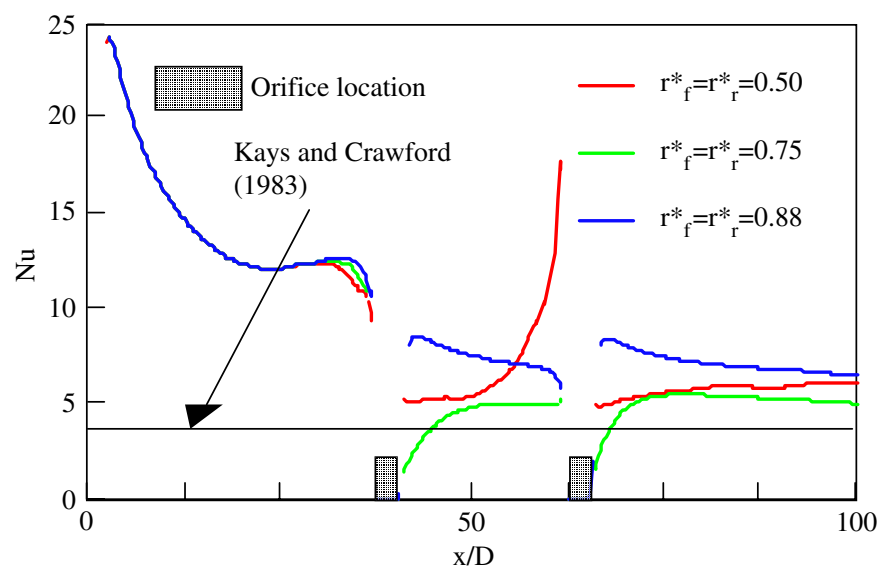

Figure 2. Effects of radius ratio on local Nusselt number for $\mathrm{Re}=480$ and $N=0$. 
number in the vena contracta is higher for both cases, i.e. for $r_{f}^{*}=0.75$ and $r_{r}^{*}=0.88$ and for $r_{f}^{*}=0.88$ and $r_{r}^{*}=0.88$ and is gradually diminished in the downstream direction. Note that the asymptotic Nusselt numbers for different rear orifice heights approach the solid line in the substantially downstream region (not shown). Throughout the numerical results, higher heat transfer performance in the region between two orifices and in the vena contracta yields for combination of radius ratios of $r_{f}^{*}=0.75$ and $r_{r}^{*}=0.88$. The heat transfer characteristics for different orifice heights become clearer for thermal and velocity profiles. Figures 4(a) and (b) depict the velocity and temperature distributions over the $r-x$ cross-section in a pipe with two concentric orifices for $r_{f}^{*}=0.75$ and $r_{r}^{*}=0.88$. Note that the passage size is substantially expanded to make the thermal and velocity fields clearer. Here, $\theta=1$ and 0 in Figure 4(b) correspond to the heated wall temperature and the fluid temperature at the inlet of the passage, respectively. One observes that as the radius ratio of the orifice is decreased, the streamwise velocity is accelerated and a recirculation zone becomes larger in the region between two orifices and behind the rear orifice. As the flow goes downstream, a thermal boundary layer develops in the sudden expansion region behind the rear orifice but its thickness is thin at the orifice with low height. This implies that the temperature gradient in the vicinity of the heated wall behind the rear orifice is larger at $r_{r}^{*}=0.88$ than at $r_{r}^{*}=0.75$, resulting in higher heat-transfer performance, as seen in Figure 3.

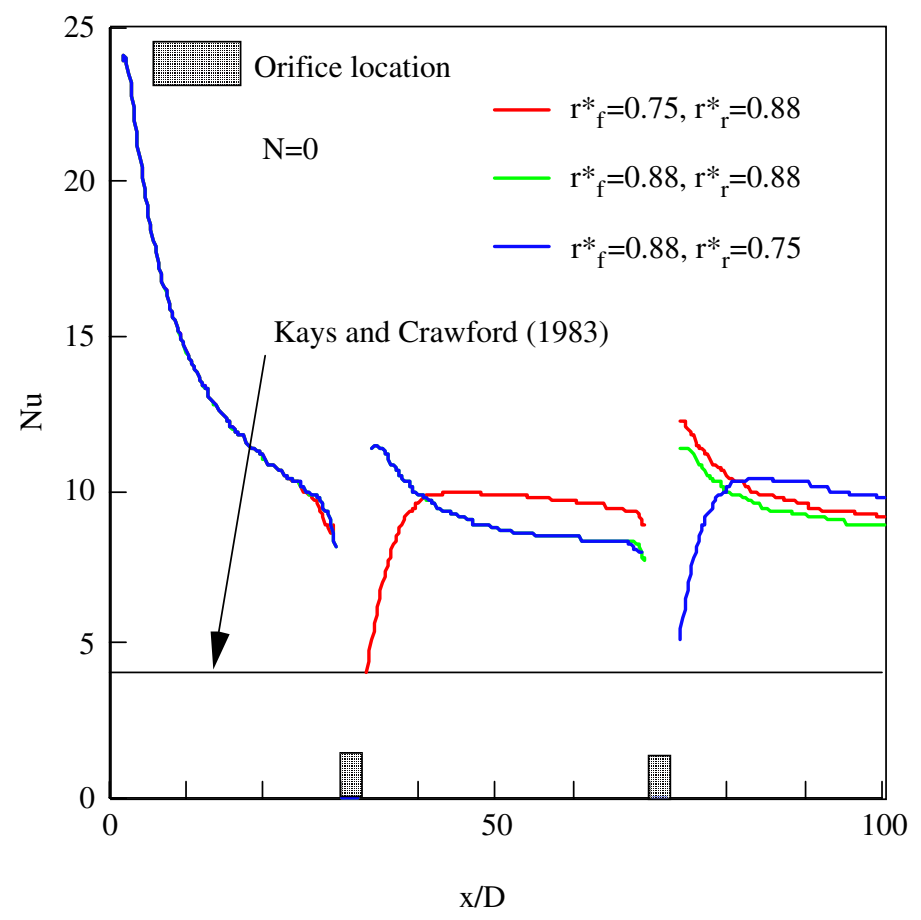

Figure 3. Effects of different radius ratios of front and rear orifices on local Nusselt numbers at $\operatorname{Re}=480$ and $N=0$. 


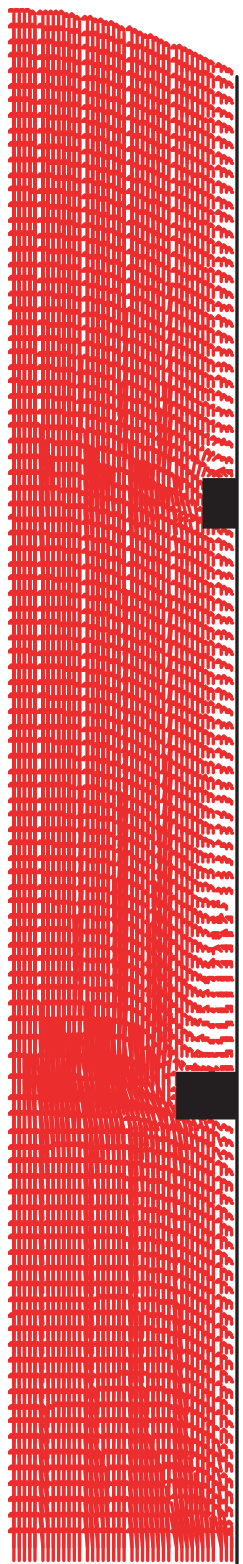

(a)

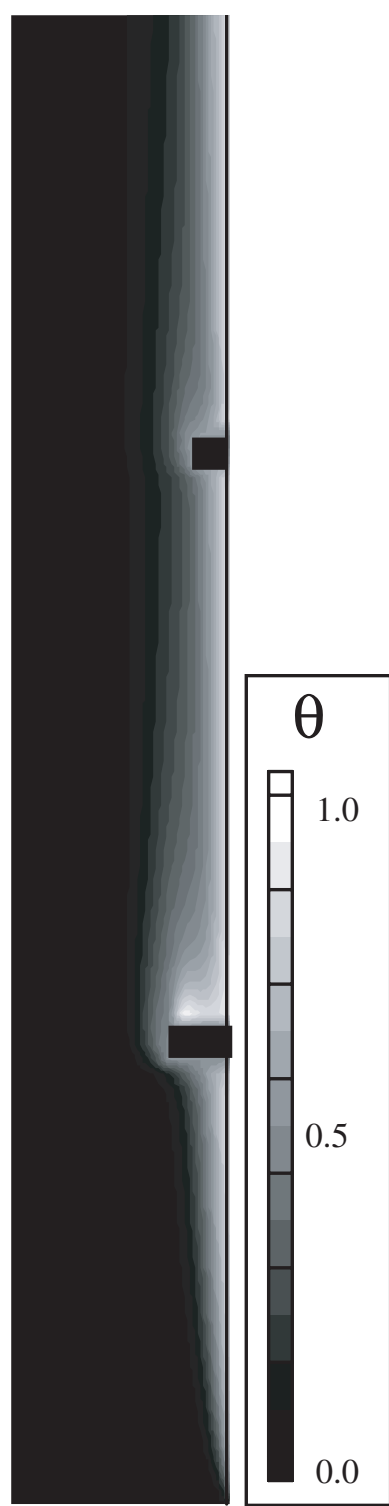

(b)

Figure 4. Velocity and thermal field in a stationary pipe with twin concentric orifices with $r_{f}^{*}=0.75$ and $r_{r}^{*}=0.88$ at $\operatorname{Re}=480$ and $N=0$ : (a) velocity vectors; and (b) temperature.

The effect of pipe rotation on the local Nusselt is depicted in Figure 5 in the same form as Figure 3. Here numerical results are obtained at $N=5$. In general, the rotation Reynolds number is employed to investigate the effects of pipe rotation and Reynolds number on the heat 


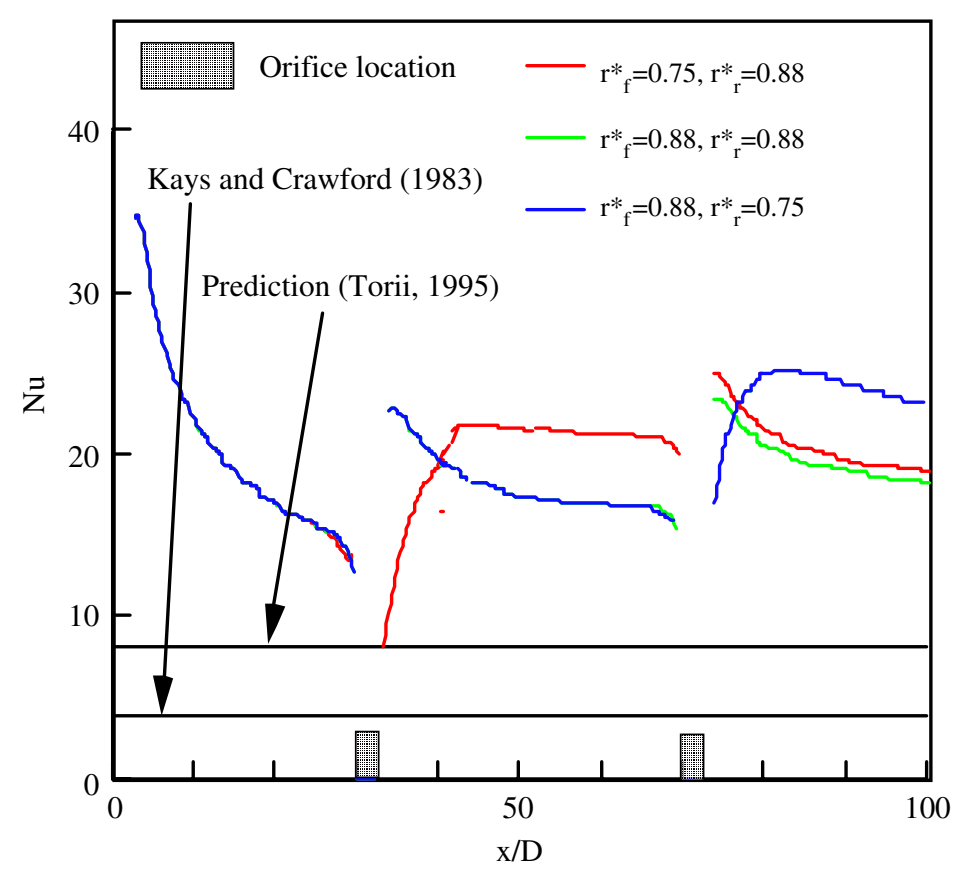

Figure 5. Effects of pipe rotation on local Nusselt numbers at $\operatorname{Re}=480$.

and thermal fields. Here, the rotation rate, $N$, is used in that the Reynolds number is fixed in the present study. No effect of radius ratio on heat-transfer performance yields in the entrance region. One observes that the substantial amplification of heat transfer performance due to pipe rotation takes places in the region between two orifices and in the vena contracta behind the rear orifice. This trend is enhanced for $r_{f}^{*}=0.75$ and $r_{r}^{*}=0.88$ and for $r_{f}^{*}=0.88$ and $r_{r}^{*}=0.75$, because the recirculation zone and isotherms are substantially stretched in the flow direction, as shown in Figures 6(a) and (b). It is observed that for $r_{f}^{*}=0.75$ and $r_{r}^{*}=0.88$, the temperature boundary layer formed in the region between two orifices is suppressed due to pipe rotation, resulting in an enhancement of temperature gradient (Figure 6(a)), and (ii) for $r_{f}^{*}=$ 0.88 and $r_{r}^{*}=0.75$, a thermal boundary layer develops in the sudden expansion region behind the rear orifice but its thickness is suppressed due to pipe rotation, resulting in an amplification of temperature gradient (Figure 6(b)). Consequently, heat transfer performance is intensified in the region between two orifices and in the vena contracta behind the rear orifice, as seen in Figure 5. Note that the asymptotic Nusselt number behind the rear orifice is the same value for three different rear orifice heights and approaches the numerical result (Torii, 1995) in the axially rotating pipe in the absence of the orifice (not shown). It is found that (i) the effect of pipe rotation on transfer performance in the region between two orifices and in the vena contracta behind the rear orifice is affected by the front and rear orifice heights and (ii) the heat transfer enhancement due to pipe rotation becomes larger when the dimensionless orifice height is 0.75 . 


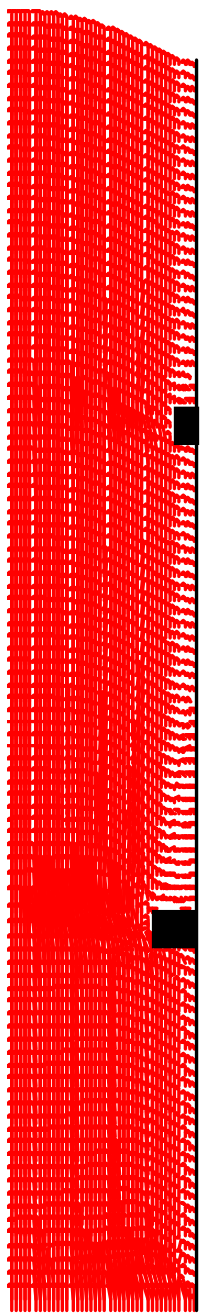

(a)

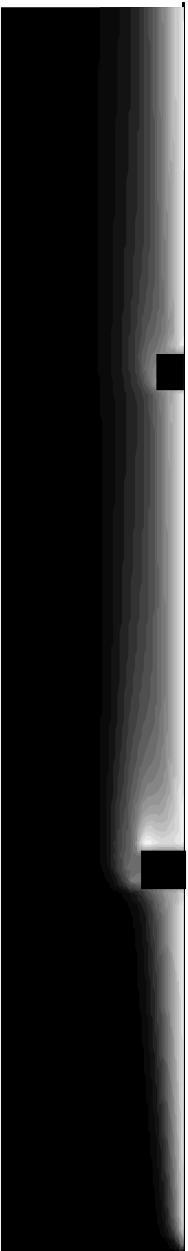

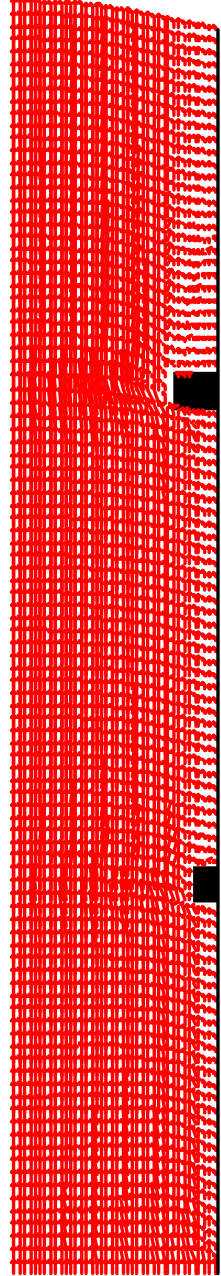

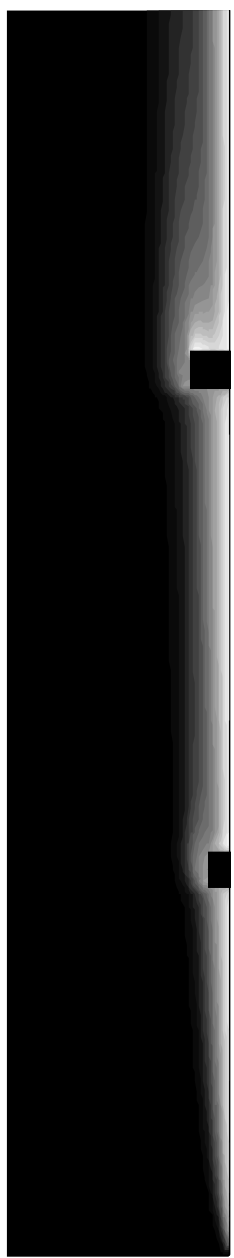

(b)

Figure 6. Velocity and temperature distributions in an axially rotating pipe at $\operatorname{Re}=480$ and $N=5$ : (a) $r_{f}^{*}=0.75$ and $r_{r}^{*}=0.88$; and (b) $r_{f}^{*}=0.88$ and $r_{r}^{*}=0.75$.

\section{CONCLUSIONS}

Numerical simulation has been employed to investigate the thermal fluid flow in an axially rotating pipe with twin concentric orifices of different heights. Consideration is given to the influence of pipe rotation and orifice-shape on the formation of a vena contracta and the heattransfer performance behind the orifice. The results are summarized as follows:

1. When laminar flow passes in a stationary pipe with the twin orifices of the same height, heat transfer enhancement in the region between two orifices and in the vena contracta is induced for $r^{*}=0.88$ than for $r^{*}=0.50$ and 0.75 . 
2. For a stationary flow with the different height front and rear orifices, higher heat transfer performance in the region between two orifices and in the vena contracta yields for combination of the radius ratio of $r_{f}^{*}=0.75$ and $r_{r}^{*}=0.88$.

3. Amplification of heat transfer performance due to pipe rotation takes places in the region between two orifices and in the vena contracta behind the rear orifice. This trend is affected by the front and rear orifice heights.

\section{NOMENCLATURE}

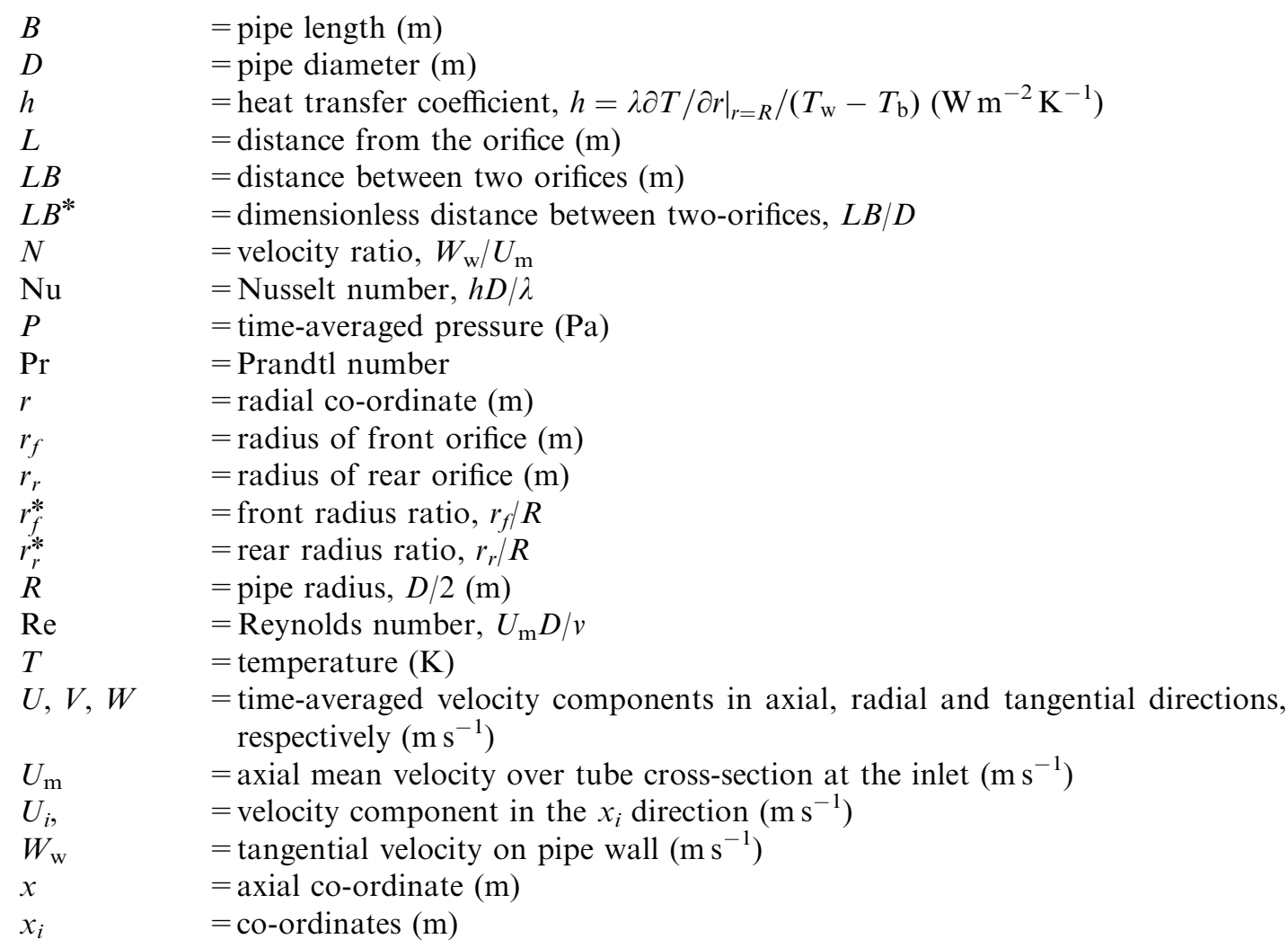

\section{Greek letters}

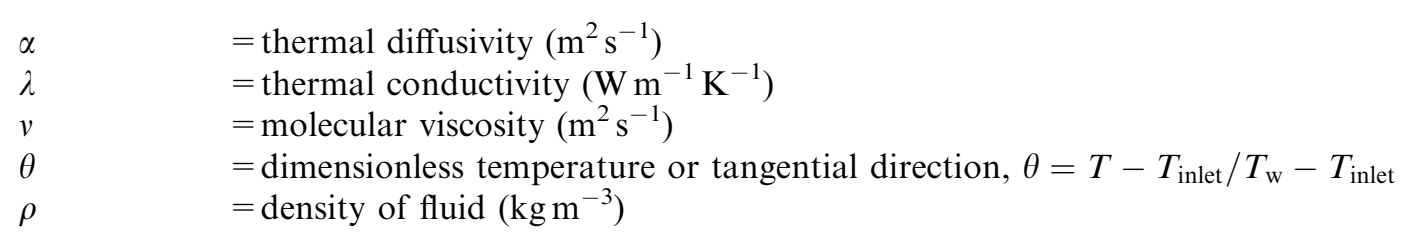




\section{THERMAL-FLUID TRANSPORT PHENOMENA}

$\begin{array}{ll}\text { Subscripts } & \\ \mathrm{b} & =\text { bulk } \\ \text { inlet } & =\text { inlet } \\ \mathrm{m} & =\text { mean } \\ \mathrm{W} & =\text { wall }\end{array}$

\section{REFERENCES}

Harlow FH, Welch EJ. 1965. Numerical calculation of time-dependent viscous incompressible flow of fluid with free surface. Physics of Fluids 8:2182-2189.

Hirt CW, Nichols BD, Romero NC. 1975. SOLA-A numerical solution algorithm for transient fluid flows. $L A S L$ Report, LA-5852.

Imao S, Zhang Q, Yamada Y. 1989. The laminar flow in the developing region of a rotating pipe. JSME International Journal of Series II 32(3):317-323.

Kays WM, Crawford ME. 1983. Convective Heat and Mass Transfer (2nd edn). McGraw-Hill: New York.

Lavan Z, Nielsen H, Fejer AA. 1969. Separation and flow reversal in swirling flows in circular ducts. Physics of Fluids 12:1747-1758.

Mizutani M, Nishibori K, Kikuyama K, Murakami M. 1987. Inlet length for laminar flow in an axially rotating pipe. Turbomachinery 15:33-40.

Torii S. 1995. A numerical prediction on fully-developed swirling flows in an axially rotating pipe by means of a modified $k-\varepsilon$ turbulence model, Proceedings of First Asian Computational Fluid Dynamics Conference, vol. 3, Hong Kong; $1049-1052$.

Torii S, Yang WJ. 1998a. Secondary flow in an axially rotating flow passage with sudden expansion or contraction. Proceedings of the 7th International Symposium on Transport Phenomena and Dynamics of Rotating Machinery, vol. C, Honolulu; 1178-1185.

Torii S, Yang WJ. 1998b. Secondary flow and heat transfer in an axially rotating flow passage with sudden expansion. Journal of Flow Visualization and Image Processing 5:115-124.

Torii S, Yang WJ. 2000. Secondary flow and heat transfer in an axially rotating flow passage with a concentric orifice. Proceedings of 8th International Symposium of Transport Phenomena and Dynamics of Rotating Machinery, vol. II, Honolulu; 821-824.

Torii S, Yang WJ, Umeda S. 1998. Flow visualization and transport phenomena over a slot-perforated flat surface between two parallel plates. Journal of Flow Visualization and Image Processing 5:63-80.

Yamada Y, Imao S. 1980. Swirling flow in an axially rotating pipe. Transactions of JSME 46:1662-1670 (in Japanese). Yamada Y, Imao S, Toyozumi K. 1985. Turbulence and its structure in an axially rotating pipe. Transactions of JSME 51:34-42 (in Japanese). 